\title{
BEREZIN QUANTIZATION OF HOMOGENEOUS BOUNDED DOMAINS
}

\author{
ANDREA LOI, ROBERTO MOSSA
}

Abstract. We prove that a homogeneous bounded domain admits a Berezin quantization.

\section{INTRODUCTION}

Let $\Omega$ be a non-compact complex manifold of dimension $n, \omega=\frac{i}{2} \partial \bar{\partial} \Phi$ a real analytic Kähler form on $\Omega, g$ the corresponding Kähler metric and $K_{\lambda}(x, y)$ ( $\lambda$ a positive constant) the reproducing kernel for the Bergman space $\mathcal{H}_{\lambda}$ of all holomorphic functions on $\Omega$ square-integrable against the measure $e^{-\lambda \Phi} \frac{\omega^{n}}{n !}$. Under the following conditions:

(1) for all suffciently large real number $\lambda$ there exists a positive constant $c_{\lambda}$ such that $K_{\lambda}(x, \bar{x})=c_{\lambda} e^{\lambda \Phi(x)}$

(2) the function

$e^{-\Phi(x, \bar{x})-\Phi(y, \bar{y})+\Phi(x, \bar{y})+\Phi(y, \bar{x})}$

is globally defined on $\Omega \times \Omega, \leq 1$ and equals 1 if and only if $x=y$, (where $\Phi(x, \bar{y})$ be a sesquianalytic extension on a neighbourhood of the diagonal in $\Omega \times \Omega$ of $\Phi)$,

F. A. Berezin [4] was able to establish a quantization procedure on $(\Omega, \omega)$. His seminal paper has inspired several interesting papers both from the mathematical and physical point of view (see, e.g., M. Engliš' work [13], for a beautiful extension of Berezin's method to complex domains which satisfiy condition (1) only asympotically and [6], 7], 8], 9] for a quantum geometric interpretation of Berezin quantization and its extension to the compact case (cfr. also the final remark at the end of the paper)).

The only known instances when the above conditions (1) and (2) are satisfied, however, are just $\Omega=\mathbb{C}^{n}$ and $\Omega$ a bounded symmetric domain (with the euclidean and the Bergman metric, respectively).

Date: November 13, 2018.

2000 Mathematics Subject Classification. 53D05; 53C55; 58F06.

Key words and phrases. Kähler metrics; Berezin quantization; bounded homogeneous domain; Calabi's diastasis function.

Research partially supported by GNSAGA (INdAM) and MIUR of Italy; the first author thank ESF for short visit grants within the program "Contact and Symplectic Topology". 
In this paper we show that these conditions are satisfied by any homogeneous bounded domain $\Omega \subset \mathbb{C}^{n}$ equipped with a homogeneous Kähler form $\omega$ (not necessarily the Bergman one). Our main result is then the following:

Theorem 1. A bounded homogeneous domain $(\Omega, \omega)$ admits a Berezin quantization.

Note that condition (1) can be expressed by saying that Rawnsley's function $\epsilon_{\lambda g}(x)=e^{-\lambda \Phi(x)} K_{\lambda}(x, \bar{x})$ (see [6]) is a positive constant for all $\lambda$ sufficiently large or, in a more recent terminology (due to S. Donaldson ([11]) for algebraic manifolds and to the first author and C. Arezzo ([2]) in the noncompact case), that the Kähler metric $g$ is balanced for all $\lambda$ large enough. Moreover, the function

$$
D_{g}(x, y):=\Phi(x, \bar{x})+\Phi(y, \bar{y})-\Phi(x, \bar{y})-\Phi(y, \bar{x})
$$

appearing in condition (2) is the so called diastasis function introduced and studied by E. Calabi in his seminal paper ([5]).

The paper consists of other two sections. In the next one we deal with assumption (1) for homogeneous bounded domain, namely we study the balanced condition for these domains. More precisely, we explicitly compute a real number $\lambda_{0}$ such that $\epsilon_{\lambda g}=c_{\lambda}$ ( $c_{\lambda}$ a positive constant) iff $\lambda \geq \lambda_{0}$. In Section 3 , after briefly recalling Berezin's quantization procedure, the definition and the main properties of Calabi's diastasis function, we show that the Kähler metric $g$ of a homogeneous bounded domain $\Omega$ satisfies condition (2). The key tool here, is the link between Rawnsley's epsilon function, Calabi's diastasis function and their relationship with the theory of Kähler immersions (following the ideas developed in [6], [7, [8] and [9]). Combining the results of Section 3 with those of Section 2 we then obtain the proof of Theorem 1 .

Acknowledgments. The authors are indebted to H. Ishi for the proof of Theorem 2 describing the structure of balanced metrics on a homogeneous bounded domain.

\section{BALANCED METRICS FOR HOMOGENEOUS BOUNDED DOMAINS}

Let $\Omega \subset \mathbb{C}^{n}$ be a complex domain of $\mathbb{C}^{n}$ endowed with a Kähler metric $g$ and let $\omega$ be the Kähler form associated to $g$, i.e. $\omega(\cdot, \cdot)=g(\cdot, J \cdot)$. Assume that the metric $g$ can be described by a strictly plurisubharmonic real valued function $\Phi: \Omega \rightarrow \mathbb{R}$, called a Kähler potential for $g$, i.e. $\omega=\frac{i}{2} \partial \bar{\partial} \Phi$.

A Kähler potential is not unique, in fact it is defined up to an addition with the real part of a holomorphic function on $\Omega$. Let $\mathcal{H}_{\Phi}$ be the weighted Hilbert space of square integrable holomorphic functions on $\Omega$, with weight $e^{-\Phi}$, namely

$$
\mathcal{H}_{\Phi}=\left\{\left.s \in \operatorname{Hol}(\Omega)\left|\int_{\Omega} e^{-\Phi}\right| s\right|^{2} \frac{\omega^{n}}{n !}<\infty\right\},
$$


If $\mathcal{H}_{\Phi} \neq\{0\}$ we can pick an orthonormal basis $\left\{s_{j}\right\}$ and define its reproducing kernel by

$$
K_{\Phi}(z, z)=\sum_{j=0}^{\infty}\left|s_{j}(z)\right|^{2} .
$$

Consider the function

$$
\varepsilon_{g}(z)=e^{-\Phi(z)} K_{\Phi}(z, z) .
$$

As suggested by the notation $\varepsilon_{g}$ depends only on the metric $g$ and not on the choice of the Kähler potential $\Phi$. In fact, if $\Phi^{\prime}=\Phi-\operatorname{Re}(\varphi)$, for some holomorphic function $\varphi$, is another potential for $\omega$, we have $e^{-\Phi^{\prime}}=$ $e^{-\Phi}\left|e^{\varphi}\right|^{2}$. Furthermore, since $\varphi$ is holomorphic and $\partial \bar{\partial} \Phi^{\prime}=\partial \bar{\partial} \Phi, e^{-} \varphi$ is an isomorphism between $\mathcal{H}_{\Phi}$ and $\mathcal{H}_{\Phi^{\prime}}$, and thus we can write $K_{\Phi^{\prime}}(z, z)=$ $\left|e^{-\varphi}\right|^{2} K_{\Phi}(z, z)$, where $K_{\Phi}(z, z)$ (resp. $\left.K_{\Phi^{\prime}}(z, z)\right)$ is the reproducing kernel of $\mathcal{H}_{\Phi}$ (resp. $\left.\mathcal{H}_{\Phi^{\prime}}\right)$. It follows that $e^{-\Phi(z)} K_{\Phi}(z, z)=e^{-\Phi^{\prime}(z)} K_{\Phi^{\prime}}(z, z)$, as claimed.

Definition. Let $g$ be a Kähler metric on a complex domain $\Omega \subset \mathbb{C}^{n}$ such that $\omega=\frac{i}{2} \partial \bar{\partial} \Phi$. The metric $g$ is balanced if the function $\varepsilon_{g}$ is a positive constant.

In the literature the function $\varepsilon_{g}$ was first introduced under the name of $\eta$-function by J. H. Rawnsley in [29], later renamed as $\varepsilon$-function in [6]. It also appears under the name of distortion function for the study of Abelian varieties by J. R. Kempf [21 and S. Ji 20, and for complex projective varieties by S. Zhang [31] (see also [16] and references therein). The definition of balanced metrics was given in the compact case by Donaldson and in the noncompact case by the first author together with C. Arezzo in [2] (see also [1]).

In this section we are interested in studying the balanced metrics when $(\Omega, g)$ is a homogeneous bounded domain, namely $\Omega \subset \mathbb{C}^{n}$ is a bounded domain, $g$ a Kähler metric on $\Omega$ and the Lie group $G=\operatorname{Aut}(\Omega) \cap \operatorname{Isom}(\Omega, g)$ acts trasitively on $\Omega$, where $\operatorname{Aut}(\Omega)$ (resp. $\operatorname{Isom}(\Omega, g)$ ) denotes the group of invertible holomorphic maps (resp. $g$-isometries) of $\Omega$. In this case, it is well-known that $\Omega$ is contractible and hence $\omega=\frac{i}{2} \partial \bar{\partial} \Phi$, for a globally defined Kähler potential $\Phi$. We start with a lemma.

Lemma 1. Let $(\Omega, g, \omega)$ be a bounded homogeneous domain, $\lambda$ a positive real number and

$$
\mathcal{H}_{\lambda}:=\mathcal{H}_{\lambda \Phi}=\left\{\left.s \in \operatorname{Hol}(\Omega)\left|\int_{\Omega} e^{-\lambda \Phi}\right| s\right|^{2} \frac{\omega^{n}}{n !}<\infty\right\} .
$$

Then $\mathcal{H}_{\lambda} \neq\{0\}$ if and only if $\lambda g$ is a balanced metric.

Proof. If $\mathcal{H}_{\lambda}=\{0\}$ then $\varepsilon_{\lambda g}$ equals the constant zero. If $\mathcal{H}_{\lambda} \neq\{0\}$ then the reproducing kernel $K_{\lambda \Phi}$ is not trivial and therefore $\varepsilon_{\lambda g}$ have to be positive at same point. For every $h \in G$ we have that $\lambda \widetilde{\Phi}(z)=\lambda \Phi \circ h$ is a Kähler 
potential for $\lambda g$ and $K_{\lambda \tilde{\Phi}}(z, \bar{z})=K_{\lambda \Phi}(h \cdot z, \overline{h \cdot z})$ is the reproducing kernel of $\mathcal{H}_{\lambda}$. Therefore

$$
\begin{aligned}
\varepsilon_{\lambda g}(z) & =e^{-\lambda \phi(z)} K_{\lambda \Phi}(z, \bar{z})=e^{-\lambda \widetilde{\phi}(z)} K_{\lambda \tilde{\Phi}}(z, \bar{z}) \\
& =e^{-\lambda \phi(h \cdot z)} K_{\lambda \Phi}(h \cdot z, \overline{h \cdot z})=\varepsilon_{\lambda g}(h \cdot z),
\end{aligned}
$$

since $G$ acts transitively on $\Omega$, we conclude that $\varepsilon_{\lambda g}$ is a positive constant.

We are now in the position to state and proof the main result of this section.

Theorem 2. Let $(\Omega, g)$ be a homogeneous bounded domain. Then there exists a positive constant $\lambda_{0}$ such that $\lambda g$ is balanced if and only if $\lambda \geq \lambda_{0}$.

Thanks to Lemma1 we are reduced to show Theorem 3 below. This theorem is known to harmonic analysis specialists and it is essentially contained in [30]. We present here a (unpublished) proof of this result due to H. Ishi who kindly gave to the authors the possibility to insert it in this paper.

Theorem 3. There exists $\lambda_{0}$ such that the Hilbert space $\mathcal{H}_{\lambda}$ is not trivial if and only if $\lambda \geq \lambda_{0}$.

Proof. By [12, Theorem 2 (c)], there exists a connected split solvable Lie subgroup $S \subset G$ acting simply transitively on the domain $\Omega$. We shall then reduce the argument to harmonic analysis on the solvable Lie group $S$, and apply the results of [30]. As first step we shall find a specific Kähler potential $\Phi$ of $\omega$ following Dorfmeister [12] (see also [10, Proof of Theorem 4]).

Taking a reference point $p_{0} \in \Omega$, we have a diffeomorphism

$$
\iota: S \ni h \stackrel{\sim}{\mapsto} h \cdot p_{0} \in \Omega,
$$

and by differentiation, we get the linear isomorphism $\mathfrak{s}:=\operatorname{Lie}(S) \ni X \stackrel{\sim}{\mapsto}$ $X \cdot p_{0} \in T_{p_{0}} M \equiv \mathbb{C}^{n}$. Then the evaluation of the Kähler form $\omega$ on $T_{p_{o}} \Omega$ is given by

$$
\omega\left(X \cdot p_{0}, Y \cdot p_{0}\right)=\beta([X, Y]) \quad(X, Y \in \mathfrak{s})
$$

with a certain linear form $\beta \in \mathfrak{s}^{*}$. Let $j: \mathfrak{s} \rightarrow \mathfrak{s}$ be the linear map defined in such a way that $(j X) \cdot p_{0}=\sqrt{-1}\left(X \cdot p_{0}\right)$ for $X \in \mathfrak{s}$. We have $g\left(X \cdot p_{0}, Y \cdot p_{0}\right)=$ $\beta([j X, Y])$ for $X, Y \in \mathfrak{s}$, and the right-hand side defines a positive inner product on $\mathfrak{s}$. Let $\mathfrak{a}$ be the orthogonal complement of $[\mathfrak{s}, \mathfrak{s}]$ in $\mathfrak{s}$ with respect to this inner product. Then $\mathfrak{a}$ is a commutative Cartan subalgebra of $\mathfrak{s}$. Define $\gamma \in \mathfrak{a}^{*}$ by

$$
\gamma(X):=-4 \beta(j X)
$$

for $X \in \mathfrak{a}$, and we extended $\gamma$ to $\mathfrak{s}=\mathfrak{a} \oplus[\mathfrak{s}, \mathfrak{s}]$ by the zero-extension. Keeping (4) in mind, we define a positive smooth function $\Psi$ on $\Omega$ by

$$
\Psi\left((\exp X) \cdot p_{0}\right)=e^{-\gamma(X)} \quad(X \in \mathfrak{s}) .
$$


From the argument in [12, pp. 302-304], we see that

$$
\omega=\frac{i}{2} \partial \bar{\partial} \log \Psi \text {. }
$$

It follows that $\Phi:=\log \Psi$ is a Kähler potential of $\omega$.

Noting that $\gamma([\mathfrak{s}, \mathfrak{s}])=0$ by definition. We define the one-dimensional representation $\chi: S \rightarrow \mathbb{R}^{+}$of $S$ by $\chi(\exp X):=e^{-\gamma(X) / 2}(X \in \mathfrak{s})$. Then (7) is rewritten as

$$
e^{-\Phi\left(h \cdot p_{0}\right)}=\chi(h)^{-2} \quad(h \in S),
$$

so that we have $e^{-\Phi(h \cdot p)}=\chi(h)^{-2} e^{-\Phi(p)}(p \in M, h \in S)$. Let $L^{2}(S)$ be the Hilbert space of square integrable functions on $S$ with respect to the left Haar measure $d g$ for which $\iota_{*} d g$ equals the $S$-invariant measure $\frac{\omega^{n}}{n !}$ on $\Omega$. Then we have the isometry $\iota^{\sharp}: \mathcal{H}_{\Phi} \rightarrow L^{2}(S)$ defined by

$$
\iota^{\sharp} F(h):=e^{-\Phi \circ \iota(h) / 2} F \circ \iota(h)=e^{-\Phi\left(h \cdot p_{0}\right) / 2} F\left(h \cdot p_{0}\right),
$$

for $h \in S$ and $F \in \mathcal{H}_{\Phi}$. We shall give an analytic description of the image of $\iota^{\sharp}$ in $L^{2}(S)$. For $X \in \mathfrak{s}$, we denote by $R(X)$ the corresponding left invariant vector field on the Lie group $S$. Namely, for $\varphi \in C^{\infty}(S)$ we have $R(X) \varphi(h):=\left.\frac{d}{d t}\right|_{t=0} \varphi(h \exp t X),(h \in S)$. For $Z=X+\sqrt{-1} Y \in \mathfrak{s} \mathbb{C}$ with $X, Y \in \mathfrak{s}$, we define $R(Z):=R(X)+\sqrt{-1} R(Y)$. Let $\mathfrak{s}_{-}$be the subspace $\{X+\sqrt{-1} j X ; X \in \mathfrak{s}\}$ of $\mathfrak{s}_{\mathbb{C}}$. Then we have a linear isomorphism $\mathfrak{s}_{-} \ni$ $Z \mapsto Z \cdot p_{0} \in T_{p_{0}}^{(0,1)} \Omega$, so that the push-forward $\iota_{*} R(Z)$ is an $S$-invariant anti-holomorphic vector field on the complex domain $\Omega$ for each $Z \in \mathfrak{s}_{-}$. Thus $\mathfrak{s}_{-}$is a complex Lie subalgebra of $\mathfrak{s}_{\mathbb{C}}$.

Let $f$ denote the linear form $-2 \beta$ on $\mathfrak{s}$. We now pause to prove the following proposition.

Proposition 2. (i) The subalgebra $\mathfrak{s}_{-} \subset \mathfrak{s}_{\mathbb{C}}$ is a positive polarization at $f$, that is, $f\left(\left[\mathfrak{s}_{-}, \mathfrak{s}_{-}\right]\right)=0$ and $\sqrt{-1} f([Z, \bar{Z}]) \geq 0$ for all $Z \in \mathfrak{s}_{-}$.

(ii) The image of $\iota^{\sharp}$ equals the function space 1

$$
\mathcal{L}(S, f):=\left\{\varphi \in L^{2}(S) ; R(Z) \varphi=-\sqrt{-1} f(Z) \varphi \text { for all } Z \in \mathfrak{s}_{-}\right\} .
$$

Proof. (i) For $Z, Z^{\prime} \in \mathfrak{s}_{-}$, we have by (5)

$$
f\left(\left[Z, Z^{\prime}\right]\right)=-2 \omega\left(Z \cdot p_{0}, Z^{\prime} \cdot p_{0}\right)=0
$$

because $\omega$ is $(1,1)$-form. Similarly, we have for $Z=X+\sqrt{-1} j X \in \mathfrak{s}_{-}$with $X \in \mathfrak{s}$,

$$
\sqrt{-1} f([Z, \bar{Z}])=2 \sqrt{-1} \omega\left(\bar{Z} \cdot p_{0}, Z \cdot p_{0}\right)=4 g\left(X \cdot p_{0}, X \cdot p_{0}\right) / 2 \geq 0 .
$$

(ii) We take $\varphi=\iota^{\sharp} F \in$ Image $\iota^{\sharp}$ with $F \in L_{h o l}^{2}\left(M, e^{-\Phi} \frac{\omega^{n}}{n !}\right)$. By (9) and (10), we obtain

$$
\varphi(h)=\chi(h)^{-1} F(\iota(h)),(h \in G) .
$$

\footnotetext{
$1_{\text {The function space }} \mathcal{L}(S, f)$ is fundamental in theory of holomorphic induction, which is closely related to Kostant's geometric quantization 3 .
} 
By the Leibniz rule, we have for $Z=X+\sqrt{-1} j X \in \mathfrak{s}_{-}(X \in \mathfrak{s})$

$$
R(Z) \varphi=R(Z) \chi^{-1} \cdot F \circ \iota+\chi^{-1} \cdot R(Z) F \circ \iota .
$$

Since $F$ is holomorphic, we have $R(Z) F \circ \iota=\iota_{*} R(Z) F=0$. Noting that $\chi^{-1 / 2}$ is a one-dimensional representation, we have

$$
R(X) \chi^{-1}=\frac{\gamma(X)}{2} \chi^{-1}=f(j X) \chi^{-1}
$$

because

$$
\gamma(X)=-4 \beta(j X)=2 f(j X) .
$$

Indeed, we may assume that (66) holds for all $X \in \mathfrak{s}_{-}$([30, Page 362]). Then $R(j X) \chi^{-1}=-f(X) \chi^{-1}$, so that $R(Z) \chi^{-1}=-\sqrt{-1} f(Z) \chi^{-1}$. Thus we have Image $\iota^{\sharp} \subset \mathcal{L}(S, f)$. The converse inculsion can be shown similarly.

By Proposition 2 (ii), the non-vanishing condition of $\mathcal{H}_{\Phi}$ is equivalent to the one of $\mathcal{L}(S, f)$, and the latter is completely determined by 30 . Theorem 4.26]. In order to apply the results in 30, we need a root structure of the Lie algebra $\mathfrak{s}$ with respect to $\mathfrak{a}$ due to Piatetskii-Shapiro 28. For a linear form $\alpha$ on the Cartan algebra $\mathfrak{a}$, we denote by $\mathfrak{s}_{\alpha}$ the subspace $\{X \in \mathfrak{s} ;[C, X]=\alpha(C) X(\forall C \in \mathfrak{a})\}$ of $\mathfrak{s}$. We say that $\alpha$ is a root if $\mathfrak{s}_{\alpha} \neq\{0\}$ and $\alpha \neq 0$. Thanks to [28, Chapter 2, Section 3] or [30, Theorem 4.3], there exists a basis $\left\{\alpha_{1}, \ldots, \alpha_{r}\right\},(r:=\operatorname{dim} \mathfrak{a})$ of $\mathfrak{a}^{*}$ such that every root is one of the following:

$$
\alpha_{k}, \alpha_{k} / 2,(k=1, \ldots, r), \quad\left(\alpha_{l} \pm \alpha_{k}\right) / 2,(1 \leq k<l \leq r) .
$$

If $\left\{A_{1}, \ldots, A_{r}\right\}$ is the basis of $\mathfrak{a}$ dual to $\left\{\alpha_{1}, \ldots, \alpha_{r}\right\}$, then $\mathfrak{s}_{\alpha_{k}}=\mathbb{R} j A_{k}$. Thus $\mathfrak{s}_{\alpha_{k}}(k=1, \ldots, r)$ is always one dimensional, whereas other root spaces $\mathfrak{s}_{\alpha_{k} / 2}$ and $\mathfrak{s}_{\left(\alpha_{l} \pm \alpha_{k}\right) / 2}$ may be $\{0\}$. We put for $k=1, \ldots, r$

$$
p_{k}:=\sum_{i<k} \operatorname{dim} \mathfrak{s}_{\left(\alpha_{k}-\alpha_{i}\right) / 2}, \quad q_{k}:=\sum_{l>k} \operatorname{dim} \mathfrak{s}_{\left(\alpha_{l}-\alpha_{k}\right) / 2}, \quad b_{k}:=\frac{1}{2} \operatorname{dim} \mathfrak{s}_{\alpha_{k} / 2},
$$

see [30, Definition 4.7] and [27, (2.7)].

Since $\left\{\alpha_{1}, \ldots, \alpha_{r}\right\}$ is a basis of $\mathfrak{a}^{*}$, the linear form $\gamma$ is written as $\gamma=$ $\sum_{k=1}^{r} \gamma_{k} \alpha_{k}$ with unique $\gamma_{1}, \ldots, \gamma_{r} \in \mathbb{R}$, where $\alpha_{k}$ 's are extended to $\mathfrak{s}$ by the zero-extension. Since $j A_{k} \in \mathfrak{s}_{\alpha_{k}}$, we obtain

$$
\begin{aligned}
\gamma_{k} & =\gamma\left(A_{k}\right)=-4 \beta\left(j A_{k}\right)=-4 \beta\left(\left[A_{k}, j A_{k}\right]\right)=4 \beta\left(\left[j A_{k}, A_{k}\right]\right) \\
& =4 g\left(A_{k} \cdot p_{0}, A_{k} \cdot p_{0}\right)>0 .
\end{aligned}
$$

By (11), we have $f=-\gamma \circ j / 2=\sum_{k=1}^{r}\left(-\gamma_{k} / 2\right) \alpha_{k} \circ j$. Now we get the following from 30 .

Proposition 3 ([30, Theorem 4.26]). The Hilbert space $\mathcal{L}(S, f) \neq 0$ if and only if

$$
\gamma_{k}>1+p_{k}+b_{k}+q_{k} / 2,(k=1, \ldots, r) .
$$


The proof of Theorem 3 follows now easily by the proof of Proposition 3 by replacing the Hilbert space $\mathcal{H}_{\Phi}$ by $\mathcal{H}_{\lambda}=\mathcal{H}_{\lambda \Phi}$ and by setting

$$
\lambda_{0}:=\max _{1 \leq k \leq r} \frac{1+p_{k}+b_{k}+q_{k} / 2}{\gamma_{k}} .
$$

Example 4. If $\omega$ is the Kähler form corresponding to the Bergman metric on $\Omega$, then $\gamma_{k}=2+p_{k}+q_{k}+b_{k}(k=1, \ldots, r)$ (see [15, Theorem 5.1] or [27. (2.19)]), so that

$$
\lambda_{0}=\max _{1 \leq k \leq r} \frac{1+p_{k}+b_{k}+q_{k} / 2}{2+p_{k}+q_{k}+b_{k}}
$$

which is found in [27, Page 97]. In particular, if $\Omega$ is a bounded symmetric domain, then there exists integers $a$ and $b$ so that

$$
p_{k}=(k-1) a, \quad q_{k}=(r-k) a, \quad b_{k}=b, \quad \gamma_{k}=(r-1) a+b+2 .
$$

Therefore

$$
\begin{aligned}
\lambda_{0} & =\max _{1 \leq k \leq r} \frac{1+(k-1) a+b+(r-k) a / 2}{\gamma}=\frac{1+(r-1) a+b}{\gamma} \\
& =\frac{\gamma-1}{\gamma},
\end{aligned}
$$

which is consistent with [26, Theorem 1].

Remark 5. Since a balanced metric is projectively induced (see e.g. [6] and [26] or the proof of Theorem 1 in the next section), it is natural to ask for which $\lambda \in \mathbb{R}^{+}$the metric $\lambda g$ is projectively induced. This problem was addressed and solved in [25, Theorem 2] for bounded symmetric domains and in [10, Theorem 4] for the more general case of homogeneous bounded domains considered in the present paper. For completeness we briefly recal here the results obtained in [10]. The crucial point is that the homogeneous metric $\lambda g$ on a homogeneous bounded domain is projectively induced if and only if the analytic extension $e^{\lambda \Phi(z, \bar{w})}$ of $e^{\lambda \Phi(z)}$ is the reproducing kernel for an Hilbert space. The condition for $e^{\lambda \Phi(z, \bar{w})}$ to be a reproducing kernel is described in [18. Then Theorem 15 in [19] tells us that

$$
\left\{0, c_{0}\right\} \cup\left(c_{0},+\infty\right) \subset W(g) \cup\{0\} \subset\left\{\frac{q_{k}}{2 \gamma_{k}} ; k=1, \ldots, r\right\} \cup\left(c_{0},+\infty\right),
$$

where $c_{0}:=\max \left\{\frac{q_{k}}{2 \gamma_{k}} ; k=1, \ldots, r\right\}$. Thefore $W(g)$ consists of a continuous part and of a discrete part with at most $r$ elements. It is interesting to note that in general the constant $c_{0}$ is different from the constant $\lambda_{0}$ in Theorem 2. This implies for example that, on any homogeneous bounded domain, there exist infinite (homothetic) projectively induced metrics which are not balanced. 


\section{Berezin quantization And the proof of Theorem 1}

Let $(\Omega, \omega)$ be a symplectic manifold and let $\{\cdot, \cdot\}$ be the associated Poisson bracket. A Berezin quantization (we refer to 4 for details) on $\Omega$ is given by a family of associative algebras $\mathcal{A}_{\hbar}$ where the parameter $\hbar$ (which plays the role of Planck constant) range over a set $E$ of positive reals with limit point 0 . Then in the direct sum $\oplus_{h \in E} \mathcal{A}_{h}$ with component-wise product $*$, one chooses a subalgebra $\mathcal{A}$, such that for an arbitrary element $f=f(\hbar) \in \mathcal{A}$, where $f(\hbar) \in \mathcal{A}_{\hbar}$, there exists a $\operatorname{limit}_{\hbar \rightarrow 0} f(\hbar)=\varphi(f) \in \lim ^{\infty}(\Omega)$. The following correspondence principle must hold: for $f, g \in \mathcal{A}$

$$
\varphi(f * g)=\varphi(f) \varphi(g), \quad \varphi\left(\hbar^{-1}(f * g-g * f)\right)=i\{\varphi(f), \varphi(g)\} .
$$

Moreover, for any pair of points $x_{1}, x_{2} \in \Omega$ there exists $f \in \mathcal{A}$ such that $\varphi(f)\left(x_{1}\right) \neq \varphi(f)\left(x_{2}\right)$.

Consider now a real analytic Kähler manifold $\Omega$, with Kähler metric $g$ and associated Kähler form $\omega$. Assume that there exists a (real analytic) global Kähler potential $\Phi: \Omega \rightarrow \mathbb{R}$. This function extends to a sesquianalytic function $\Phi(x, \bar{y})$ on a neighbourhood of the diagonal in $\Omega \times \Omega$ such that $\Phi(x, \bar{x})=\Phi(x)$. Consider Calabi's diastasis function $D_{g}$ defined on a neighbourhood of the diagonal in $\Omega \times \Omega$ by:

$$
D_{g}(x, y)=\Phi(x, \bar{x})+\Phi(y, \bar{y})-\Phi(x, \bar{y})-\Phi(y, \bar{x}) .
$$

By its definition we see that Calabi's diastasis function is independent from the potential chosen which is defined up to the sum with the real part of a holomorphic function. Moreover, it is easily seen that $D_{g}$ is real-valued, symmetric in $x$ and $y$ and $D_{g}(x, x)=0$ (the reader is referred to [5] and 24] for more details on the diastasis function).

Example 6. Let $g_{F S}$ be the Fubini-Study metric on the infinite dimensional complex proective space $\mathbb{C} P^{\infty}$ of holomorphic sectional curvature 4 and let $D_{g_{F S}}(p, q)$ be the associated Calabi's diastasis function. One can show that for all $p \in \mathbb{C} P^{\infty}$ the function $D_{g_{F S}}(p, \cdot)$ is globally defined except in the cut locus $H_{p}$ of $p$ where it blows up. Moreover $e^{-D_{g_{F S}}(p, q)}$ is globally defined and smooth on $\mathbb{C} P^{\infty}, e^{-D_{g_{F S}}(p, q)} \leq 1$ and $e^{-D_{g_{F S}}(p, q)}=1$ if and only if $p=q$ (see [24] for details).

The following theorem is a reformulation of Berezin quantization result (see Engliš [13]) in terms of Rawnsley $\varepsilon$-function and Calabi's diastasis function.

Theorem 4. Let $\Omega \subset \mathbb{C}^{n}$ be a complex domain equipped with a real analytic Kähler form $\omega$ and corresponding Kähler metric $g$. Then, $(\Omega, \omega)$ admits a Berezin quantization if the following two conditions are satisfied:

(1) Rawnsley's function $\varepsilon_{\lambda g}(x)$ is a positive constant for all sufficiently large $\lambda$; 
(2) the function $e^{-D_{g}(x, y)}$ is globally defined on $\Omega \times \Omega, e^{-D_{g}(x, y)} \leq 1$ and $e^{-D_{g}(x, y)}=1$, if and only if $x=y$,

We are now in the position to prove Theorem 1, namely that a bounded homogeneous domain admits a Berezin quantization.

Proof of Theorem 1. Condition (1) in Theorem 4 is a consequence of Theorem 2 of the previous section. Hence, it remains to show that (2) in Theorem 4 is satisfied by a homogeneous bounded domain $(\Omega, \omega, g)$ 2. Fix $\lambda \geq \lambda_{0}$ with $\lambda_{0}$ given by Theorem 2. Consider the coherent states map (see [29]), namely the holomorphic map $\varphi_{\lambda}: \Omega \rightarrow \mathbb{C} P^{\infty}$ from $\Omega$ into the infinite dimensional complex projective space $\mathbb{C} P^{\infty}$ defined by

$$
\varphi_{\lambda}: \Omega \rightarrow \mathbb{C} P^{\infty}, x \mapsto\left[s_{0}^{\lambda}(x), \ldots, s_{j}^{\lambda}(x), \ldots\right],
$$

where $\left\{s_{j}^{\lambda}\right\}_{j=0, \ldots}$ is an orthonormal basis of the Hilbert space $\mathcal{H}_{\lambda}$ given by (3). This map is well-defined since $\epsilon_{\lambda g}$ is a positive constant and hence, for a given $x \in \Omega$, there exists $j_{0}$ such that $s_{j_{0}}$ does not vanish at $x$. Moreover, the constancy of $\varepsilon_{\lambda g}$ implies that $\varphi_{\lambda}^{*} g_{F S}=\lambda g$, where $g_{F S}$ is the FubiniStudy metric on $\mathbb{C} P^{\infty}$ (see [29] for a proof). In other words, the metric $\lambda g$ is projectively induced via the coherent states map. By Example 6, Calabi's diastasis function $D_{g_{F S}}$ of $\mathbb{C} P^{\infty}$ is such that $e^{-D_{F S}}$ is globally defined on $\mathbb{C} P^{\infty} \times \mathbb{C} P^{\infty}$ and by the hereditary property of the diastasis function (see [5. Proposition 6 ]) we get that, for all $x, y \in \Omega$,

$$
e^{-D_{F S}\left(\varphi_{\lambda}(x), \varphi_{\lambda}(y)\right)}=e^{-D_{\lambda g}(x, y)}=e^{-\lambda D_{g}(x, y)}=\left(e^{-D_{g}(x, y)}\right)^{\lambda}
$$

is globally defined on $\Omega \times \Omega$. Since, by Example 6), $e^{-D_{F S}(p, q)} \leq 1$ for all $p, q \in \mathbb{C} P^{\infty}$ it follows that $e^{-D_{g}(x, y)} \leq 1$ for all $x, y \in \Omega$. It remains to show that $e^{-D_{g}(x, y)}=1$ iff $x=y$. By formula (13) and by the fact that $e^{-D_{F S}(p, q)}=1$ iff $p=q$ (again by Example 6) this is equivalent to the injectivity of the coherent states map $\varphi_{\lambda}$. This, in turns, follows by a recent result [10, Theorem 3] of the first author together with A. J. Di Scala and H. Ishi which asserts that a Kähler immersion of a homogeneous Kähler manifold (not necessarily a bounded domain) into a finite or infinite dimensional complex projective space is one to one.

Final remark All the results of this paper can be formulated in term of geometric quantization tools, i.e. holomorphic line bundle, coherent states, charactersitic 2-point function as in [6], [7, [8] and [9]. For a bounded homogeneous domain $(\Omega, g, \omega)$ the quantum line bundle, namely the holomorphic line bundle over $\Omega$ whose first Chern class equals the De Rham class of $\omega$, is trivial and so its global holomorphic sections can be identified with the holomorphic functions on $\Omega$. A natural problem one could tries

\footnotetext{
${ }^{2}$ It is well-known that $g$ is real analytic so it makes sense to verify (2).
} 
to solve is that of obtaining a quantization by deformation (or equivalently a $*$-product) of a bounded homogeneous domain starting from the Berezin quantization given by Theorem 1 and following the ideas developed in 9] for the case of bounded symmetric domains. This is not a straightforward matter. Indeed in 9] Cahen, Gutt and Rawnsley use the polydisk theorem while for a general homogeneous bounded domain no such theorem seems to be avaliable. Moreover, to the authors best knowledge, a classification of all bounded homogeneous domains is still missing. We will attack this problem in a forthcoming paper. We finally point out that in [7] Cahen, Gutt and Rawnsley prove the existence of a Berezin $*$-product on any compact flag manifold. The results obtained by Cahen, Gutt and Rawnsley for the compact case (see in particular [7, Proposition 3] which represents the analogous of our Theorem 1 and Theorem 2) strongly rely on Kodaira's theory for projective algebraic manifolds which can not be apply to the noncompact context.

\section{REFERENCES}

[1] C. Arezzo and A. Loi, Quantization of Kähler manifolds and the asymptotic expansion of Tian-Yau-Zelditch, J. Geom. Phys. 47 (2003), 87-99.

[2] C. Arezzo and A. Loi, Moment maps, scalar curvature and quantization of Kähler manifolds, Comm. Math. Phys. 246 (2004), 543-549.

[3] L. Auslander, B. Kostant, Polarization and unitary representations of solvable Lie groups, Invent. Math. 14 (1971), 255-354.

[4] F. A. Berezin, Quantization, Math. USSR Izvestiya 8 (1974), 1109-1163. MR 52:16404.

[5] E. Calabi, Isometric Imbeddings of Complex Manifolds, Ann. of Math. 58 (1953), $1-23$.

[6] M. Cahen, S. Gutt, J. H. Rawnsley, Quantization of Kähler manifolds I: Geometric interpretation of Berezin's quantization, JGP. 7 (1990), 45-62.

[7] M. Cahen, S. Gutt, J. H. Rawnsley, Quantization of Kähler manifolds II, Trans. Amer. Math. Soc. 337 (1993), 73-98.

[8] M. Cahen, S. Gutt, J. H. Rawnsley, Quantization of Kähler manifolds III, Lett. Math. Phys. 30 (1994), 291-305.

[9] M. Cahen, S. Gutt, J. H. Rawnsley, Quantization of Kähler manifolds IV, Lett. Math. Phys. 34 (1995), 159-168.

[10] A. J. Di Scala, H. Ishi and A. Loi, Kaehler immersions of homogeneous Kaehler manifolds into complex space forms, arXiv:1009.4045v1.

[11] S. Donaldson, Scalar Curvature and Projective Embeddings, I, J. Diff. Geometry 59 (2001), 479-522.

[12] J. Dorfmeister, Simply transitive groups and Kähler structures on homogeneous Siegel domains, Trans. Amer. Math. Soc. 288 (1985), 293-305.

[13] M. Engliš Berezin Quantization and Reproducing Kernels on Complex Domains, Trans. Amer. Math. Soc. vol. 348 (1996), 411-479.

[14] M. Engliš Weighted Bergman kernels and balanced metrics, RIMS Kokyuroku 1487 (2006), 40-54.

[15] S. G. Gindikin, Analysis in homogeneous domains, Russian Math. Surveys 19 (1964), $1-89$.

[16] T. Gramchev and A. Loi, TYZ expansion for the Kepler manifold, Comm. Math. Phys. 289, (2009), 825-840.

[17] S. Helgason, Differential geometry and symmetric spaces, Academic Press, New York, 1962. MR 26:2986 
[18] H. Ishi, Representations of the affine transformation groups acting simply transitively on Siegel domains, J. Funct. Anal. 167 (1999), no. 2, 425-462.

[19] H. Ishi, Unitary holomorphic multiplier representations over a homogeneous bounded domain, to appear in Adv. Pure Appl. Math.

[20] S. Ji, Inequality for distortion function of invertible shaves on Abelian varieties, Duke Math. J. 58 (1989), 657-667.

[21] G. R. Kempf, Metrics on invertible shaves on abelian varieties, Topics in algebraic geometry (Guanajuato, 1989), Aportationes Mat. Notas Investigacion 5, Soc. MAt. Mexicana, Mexico, 1992, pp. 107-108.

[22] A. Greco, A. Loi, Radial balanced metrics on the unit disk J. Geom. Phys. 60 (2010), 53-59.

[23] A. Loi, Regular quantizations of Kähler manifolds and constant scalar curvature metrics, J. Geom. Phys. 53 (2005), 354-364.

[24] A. Loi, Calabi's diastasis function for Hermitian symmetric spaces, Diff.l Geom. Appl. 24 (2006), 311-319.

[25] A. Loi, M. Zedda, Kähler-Einstein submanifolds of the infinite dimensional projective space, Math. Ann. 350 (2011), 145-154.

(joint with M. Zedda) Balanced metrics on Hartogs domains to appear in Abh. Math. Sem. Univ. Hamburg (2011).

[26] A. Loi, M. Zedda, Balanced metrics on Cartan and Cartan-Hartogs domain, preprint, arXiv1010.1034.

[27] T. Nomura Berezin transforms and Laplace-Beltrami operators on homogeneous Siegel domains, J. Lie Theory 11 (2001), 185-206.

[28] I. I. Piatetskii-Shapiro, Automorphic functions and the geometry of classical domains, Gordon and Breach, New York, 1969.

[29] J. Rawnsley, Coherent states and Kähler manifolds, Quart. J. Math. Oxford (2), n.28 (1977), 403-415.

[30] H. Rossi, M. Vergne, Representations of certain solvable Lie groups on Hilbert spaces of holomorphic functions and the application to the holomorphic discrete series of a semisimple Lie group, J. Functional Analysis 13 (1973), 324-389.

[31] S. Zhang, Heights and reductions of semi-stable varieties, Comp. Math. 104 (1996), 77-105.

Dipartimento di Matematica e Informatica, Università di Cagliari, Via OsPEDALE 72, 09124 CAGLIARI, ITALY

E-mail address: loi@unica.it; roberto.mossa@gmail.com 